\title{
A multivariate Wald-Wolfowitz rank test against serial dependence
}

\author{
Marc HALLIN and Madan L. PURI* \\ Université Libre de Bruxelles and Indiana University
}

Key words and phrases: Wald-Wolfowitz rank test, rank cross-covariance matrix, multivariate ARMA models, multivariate portmanteau test, multivariate model identification. AMS 1985 subject classifications: Primary 62M10, 62G10.

\section{ABSTRACT}

Rank-based cross-covariance matrices, extending to the case of multivariate observed series the (univariate) rank autocorrelation coefficients introduced by Wald and Wolfowitz (1943), are considered. A permutational central limit theorem is established for the joint distribution of such matrices, under the null hypothesis of (multivariate) randomness as well as under contiguous alternatives of (multivariate) ARMA dependence. A rank-based, permutationally distribution-free test of the portmanteau type is derived, and its asymptotic local power is investigated. Finally, a modified rank-based version of Tiao and Box's model specification procedure is proposed, which is likely to be more reliable under non-Gaussian conditions, and more robust against gross errors.

\section{RÉSUMÉ}

Des matrices de covariances croisées fondées sur les rangs, généralisant au cas des séries multivariées les coefficients d'autocorrélation de rangs introduits par Wald et Wolfowitz (1943) sont considérées. Un théorème central-limite permutationnel est établi pour ces matrices, sous l'hypothèse que la série sous-jacente constitue la réalisation d'un bruit blanc multivarié, ainsi que sous des contre-hypothèses contiguës de dépendance ARMA. Un test de rangs du type portemanteau est également construit, et sa puissance asymptotique locale est explicitement calculée. Enfin, une version fondée sur les rangs de la procédure d'identification de Tiao et Box est proposée. Celle-ci est plus fiable que la procédure usuelle sous des conditions non gaussiennes, et plus robuste par rapport à la présence de valeurs aberrantes.

\section{RANK-BASED CROSS-COVARIANCE MATRICES OF THE SPEARMAN-WALD-WOLFOWITZ TYPE}

Denote by $\mathbf{X}^{(n)}=\left(\mathbf{X}_{1}^{(n)}, \ldots, \mathbf{X}_{t}^{(n)}, \ldots, \mathbf{X}_{n}^{(n)}\right)$ a series of $m$-variate observations $\mathbf{X}_{t}^{(n)}=$ $\left(X_{t, l}^{(n)}, \ldots, X_{t, m}^{(n)}\right)^{\top}, t=1, \ldots, n$. We are interested in the null hypothesis $\mathcal{H}^{(n)}$ of randomness under which the observations $\mathbf{X}_{f}^{(n)}$ are independently and identically distributed, according to some unspecified density $f(\mathbf{x}), \mathbf{x}=\left(x_{1}, \ldots, x_{m}\right)^{\top} \in \mathbb{R}^{m}$. Whenever $f$ needs to be specified, the resulting subhypothesis will be denoted by $\mathcal{H}_{f}{ }^{(n)}$. The alternatives we are considering are serial-dependence alternatives, with emphasis on multivariate ARMA dependence.

Much attention has been devoted recently to the one-dimensional version of this nonparametric problem; see, e.g., Dufour (1981), Dufour and Roy $(1985,1986)$, Chan and Tran (1992) or Shaug and Tjøstheim (1993). In this univariate version $[m=1$; observed 
series $\mathbf{X}^{(n)}=\left(X_{1}^{(n)}, \ldots, X_{n}^{(n)}\right)$ ], usual invariance arguments lead to the consideration of rank-based testing procedures; see Hallin and Puri (1992). In this latter context, Wald and Wolfowitz (1943) proposed test statistics of the form (up to additive and multiplicative constants) $S_{i}^{(n)}=n^{-1} \sum_{t=1}^{n} R_{t}^{(n)} R_{t-i}^{(n)}$, where $R_{t}^{(n)}$ denotes, as usual, the rank of $X_{t}^{(n)}$ among $X_{1}^{(n)}, \ldots, X_{n}^{(n)}$, and with the (convenient but rather arbitrary) convention that $R_{s}^{(n)}, s=0$, $-1, \ldots,-i+1$, is set equal to $R_{n+s}^{(n)}$. Clearly, $S_{i}^{(n)}$ is a (circular) serial version, of lag $i$, of Spearman's ordinary rank correlation coefficient (still, up to additive and multiplicative constants). The performance of such test statistics in a time series context have been investigated by Knoke (1977) and Bartels (1982), among others - see also Hallin and Mélard (1988). A systematic theoretical study of serial, rank-based statistics is provided in Hallin et al. $(1985,1987)$ and Hallin and Puri $(1988,1991,1994)$.

Our objective here is to extend Wald and Wolfowitz's idea to the problem of testing for multivariate randomness. Due to the greater complexity inherent in the nature of multivariate variables, the development of multivariate theories - especially in the area of rank-based procedures-typically has been much slower than that of their univariate counterparts. Multivariate rank-based statistics for serial-dependence problems have been introduced in Hallin et al. (1989), from which several asymptotic results are used here.

The multivariate generalization of Wald and Wolfowitz's rank-based autocorrelation coefficients quite naturally takes the form of rank-based cross-covariance matrices. Letting $R_{t, i}^{(n)}$ denote the rank of $X_{f, i}^{(n)}$ among $X_{1, i}^{(n)}, \ldots, X_{n, i}^{(n)}$, put $\mathbf{R}_{f}^{(n)}=\left(R_{t, 1}^{(n)}, \ldots, R_{t, m}^{(n)}\right)^{\top}$, and $\mathbf{R}^{(n)}=\left(\mathbf{R}_{1}^{(n)}, \ldots, \mathbf{R}_{n}^{(n)}\right): \mathbf{R}^{(n)}$ is called the rank-collection matrix associated with the observed series $\mathbf{X}^{(n)}$.

It is well known that, unless $m=1$, the rank vectors $\mathbf{R}_{t}^{(n)}$ are not distribution-free, even under the null hypothesis of randomness. If however we denote by $\mathbf{R}_{*}^{(n)}$ the rankcollection matrix resulting from $\mathbf{R}^{(n)}$ on rearranging the columns in such a manner that the first row yields elements $1,2, \ldots, n$ in ascending order, then the $n$ ! possible permutations of the columns of $\mathbf{R}_{*}^{(n)}$ are conditionally equiprobable: the rank vectors $\mathbf{R}_{t}^{(n)}$ are thus conditionally distribution-free, given the "ordered" rank-collection matrix $\mathbf{R}_{*}^{(n)}$.

The consideration of this conditional distribution actually also follows from unbiasedness and Neyman-structure arguments. The matrix $\mathbf{X}_{*}^{(n)}$ resulting from $\mathbf{X}^{(n)}$ on rearranging the columns as in $\mathbf{R}_{*}^{(n)}$ indeed can be shown to be sufficient complete under $\mathcal{H}^{(n)}$. Conditioning, as usual, upon this sufficient complete statistic yields the permutational distribution of $\left(\mathbf{X}_{1}^{(n)}, \ldots, \mathbf{X}_{n}^{(n)}\right)$. Additional componentwise invariance arguments then lead to the permutational distribution of $\mathbf{R}^{(n)}$, which is precisely the conditional distribution of the rank vectors given in $\mathbf{R}_{*}^{(n)}$.

Define the rank cross-covariance matrix of lag $i$ as

$$
\Gamma_{i}^{(n)}=(n-i)^{-1} \sum_{t=i+1}^{n}\left(\mathbf{R}_{t}^{(n)}\right)\left(\mathbf{R}_{t-i}^{(n)}\right)^{\top}-\mathbf{m}_{i *}^{(n)}, \quad i \geq 1,
$$

where $m_{i *}^{(n)}$ denotes the permutational mean of $(n-i)^{-1} \sum\left(\mathbf{R}_{i}^{(n)}\right)\left(\mathbf{R}_{t-i}^{(n)}\right)^{\top}$. Denote by $\gamma_{i ; k, l}^{(n)}$ the elements of $\Gamma_{i}^{(n)}$ : for $k \neq l, \gamma_{i ; k, l}^{(n)}$ is a rank cross-covariance coefficient of lag $i$, and for $k=l$ it is a (univariate) rank autocovariance coefficient, of the type studied in Hallin et al. $(1985,1987)$. Note that a similar notation is adopted in Hallin et al. (1989) for a more general type of rank cross-covariance matrix, which we do not consider here.

Section 2 below is devoted to the asymptotic distribution theory of rank crosscovariance matrices: $(n-i)^{\frac{1}{2}}(n+1)^{-2}$ vec $\Gamma_{i}^{(n)}$ will be shown to be asymptotically multinormal, under the null hypothesis of randomness as well as under contiguous alternatives of ARMA dependence. Section 3 discusses statistical applications in time-series 
analysis. Before proceeding to asymptotic results, however, we start with an explicit expression for the permutational mean matrices $\mathbf{m}_{i *}^{(n)}$. Here 1 stands for a matrix of ones of appropriate dimensions; $O_{P}$ and $o_{P}$ are unconditional $O_{P}$ 's and $o_{P}$ 's.

PROPOSITION 1.1. The permutational mean matrices $\mathbf{m}_{i *}^{(n)}$ in (1.1) are of the form

$$
\mathbf{m}_{i *}^{(n)}=\frac{n(n+1)^{2}}{4(n-1)} \mathbf{1}-\frac{\mathbf{m}_{0 *}^{(n)}}{n-1}
$$

with $\mathbf{m}_{0 *}^{(n)}=n^{-1} \sum_{t=1}^{n}\left(\mathbf{R}_{t}^{(n)}\right)\left(\mathbf{R}_{t}^{(n)}\right)^{\top}$. Their elements uniformly satisfy

$$
\frac{n^{2}}{4}<\frac{(n+1)(3 n+2)}{12} \leq\left(\mathbf{m}_{i *}^{(n)}\right)_{k l}<\frac{n(n+1)^{2}}{4(n-1)}
$$

Moreover, as $n \rightarrow \infty$,

$$
n^{-2} \mathbf{m}_{i *}^{(n)}=\left\{\frac{1}{4}+O_{P}\left(n^{-1}\right)\right\} \mathbf{1}, \quad i \geq 1 .
$$

Proof. For $i=1,2, \ldots, n-1$, we have

$$
\begin{aligned}
\mathbf{m}_{i *}^{(n)} & =\{n(n-1)\}^{-1} \sum_{1<t_{1} \neq t_{2} \leq n}\left(\mathbf{R}_{t_{1}}^{(n)}\right)\left(\mathbf{R}_{t_{2}}^{(n)}\right)^{\top} \\
& =\{n(n-1)\}^{-1}\left(\sum_{t=1}^{n}\left(\mathbf{R}_{t}^{(n)}\right) \sum_{t=1}^{n}\left(\mathbf{R}_{t}^{(n)}\right)^{\top}-n \mathbf{m}_{0 *}^{(n)}\right) \\
& =\{n(n-1)\}^{-1}\left\{\left(\sum_{r=1}^{n} r\right)^{2} 1-n \mathbf{m}_{0 *}^{(n)}\right\} \\
& =\frac{n(n-1)^{2}}{4(n-1)} 1-\frac{\mathbf{m}_{0 *}^{(n)}}{n-1} .
\end{aligned}
$$

Now, $\left(n \mathbf{m}_{0 *}^{(n)}\right)_{k, l}=\sum_{t=1}^{n} R_{t, k}^{(n)} R_{t, l}^{(n)} \leq \sum_{t=1}^{n}\left(t^{2}\right)=n(n+1)(2 n+1) / 6 ;(1.3)$ and (1.4) straightforwardly follow.

Note that $\mathbf{m}_{i *}^{(n)}, i \geq 1$, does not depend on $i$.

\section{ASYMPTOTIC DISTRIBUTION THEORY}

Rank cross-covariance matrices can be used for testing $\mathcal{H}^{(n)}$ against arbitrary alternatives of serial dependence. If however local asymptotic power results are desired, more specific alternatives are to be considered. Denote by $\mathcal{K}_{f}^{(n)}(\mathbf{A}, \mathbf{B})$ the local alternative under which $\mathbf{X}^{(n)}$ constitutes a finite realization of some solution of the stochastic difference equation (ARMA model)

$$
\mathbf{X}_{t}-n^{-\frac{1}{2}} \sum_{i=1}^{p} \mathbf{A}_{i} \mathbf{X}_{t-i}=\boldsymbol{\varepsilon}_{t}+n^{-\frac{1}{2}} \sum_{i=1}^{q} \mathbf{B}_{i} \boldsymbol{\varepsilon}_{t-i}, \quad t \in \mathbf{Z},
$$

where $\mathbf{E}_{\boldsymbol{l}}$ is an independent $m$-variate white noise with density function $f$. Assume that $f$ satisfies some mild technical conditions, such as being twice differentiable [put $\boldsymbol{\phi}(\mathbf{x})=$ $-\operatorname{grad} \log f(\mathbf{x})]$ and having finite Fisher information $\left[\int \boldsymbol{\phi}(\mathbf{x}) \boldsymbol{\phi}^{\top}(\mathbf{x}) f(\mathbf{x}) d \mathbf{x}=\mathbf{I}(f)<\infty\right]$. 
Then the sequences $\mathcal{K}_{f}^{(n)}(\mathbf{A}, \mathbf{B})$ and $\mathcal{H}_{f}^{(n)}$ are contiguous [see Hallin et al. (1989) for details].

Denote by $F, f_{i}$ and $F_{i}(i=1, \ldots, m)$, respectively, the $m$-dimensional distribution function, marginal densities and marginal distribution functions associated with $F$. Assume that $\int \mathbf{x} f(\mathbf{x}) d \mathbf{x}=0$, and that $\int \mathbf{x x}^{\top} f(\mathbf{x}) d \mathbf{x}=\mathbf{Y}$, a finite, strictly positive definite covariance matrix. Finally, define $\mathbf{F}(\mathbf{x})=\left(F_{1}\left(x_{1}\right), \ldots, F_{m}\left(x_{m}\right)\right)^{\top}, \mathbf{x}=\left(x_{1}, \ldots, x_{m}\right)^{\top} \in \mathbb{R}^{m}$. Proposition 2.1. Let $i^{\prime} \neq i^{\prime \prime}$. As $n \rightarrow \infty$, the joint distribution of $(n+1)^{-2}\left(\left(n-i^{\prime}\right)^{\frac{1}{2}}\right.$ $\left.\operatorname{vec}^{\top} \Gamma_{i^{\prime}}^{(n)},\left(n-i^{\prime \prime}\right)^{\frac{1}{2}} \operatorname{vec}^{\top} \Gamma_{i^{\prime \prime}}^{(n)}\right)^{\top}$ is asymptotically multinormal, with mean $\mathbf{0}$ under $\mathscr{H}^{(n)}$ and mean

$$
\left(\begin{array}{l}
\left\{\int \mathbf{F}(\mathbf{x}) \mathbf{x}^{\top} d F(\mathbf{x})\right\} \otimes\left\{\int \mathbf{F}(\mathbf{x}) \boldsymbol{\phi}^{\top}(\mathbf{x}) d F(\mathbf{x})\right\} \operatorname{vec}\left(\mathbf{A}_{i^{\prime}}+\mathbf{B}_{i^{\prime}}\right) \\
\left\{\int \mathbf{F}(\mathbf{x}) \mathbf{x}^{\top} d F(\mathbf{x})\right\} \otimes\left\{\int \mathbf{F}(\mathbf{x}) \boldsymbol{\phi}^{\top}(\mathbf{x}) d F(\mathbf{x})\right\} \operatorname{vec}\left(\mathbf{A}_{i^{\prime \prime}}+\mathbf{B}_{i^{\prime \prime}}\right)
\end{array}\right)
$$

under $\mathcal{K}_{f}^{(n)}(\mathbf{A}, \mathbf{B})$, and with covariance matrix

$$
\left(\begin{array}{cc}
{\left[\int \mathbf{F}(\mathbf{x})\{\mathbf{F}(\mathbf{x})\}^{\top} d F(\mathbf{x})-\frac{1}{4}\right]^{\otimes 2}} & \mathbf{0} \\
\mathbf{0} & {\left[\int \mathbf{F}(\mathbf{x})\{\mathbf{F}(\mathbf{x})\}^{\top} d F(\mathbf{x})-\frac{1}{4}\right]^{\otimes 2}}
\end{array}\right)
$$

under both (all diagonal elements in this latter matrix are equal to $\frac{1}{144}$ ).

Proof. Letting $I=\max \left(i^{\prime}, i^{\prime \prime}\right)$, we see that $(n+1)^{-2}\left(\gamma_{j^{\prime} ; k, l}^{(n)}+m_{j^{*}: k, l}^{(n)}\right)$ is of the form

$$
(n-I)^{-1} \sum_{t=l+1}^{n} J_{k}\left(\frac{\mathbf{R}_{t}^{(n)}}{n+1}\right) J_{l}\left(\frac{\mathbf{R}_{t-j}^{(n)}}{n+1}\right)+o_{P}\left(n^{-\frac{1}{2}}\right),
$$

where $J_{h}(\mathbf{u})=u_{h}(h=1, \ldots, m)-$ a nondecreasing function with respect to all the components of $\mathbf{u} \in(0,1)^{m}$ - satisfies $\mathcal{E} J_{h}^{2}\left(\mathbf{F}\left(\mathbf{X}_{1}^{(n)}\right)\right)=\int_{0}^{1} u^{2} d u<\infty$. The assumptions of Proposition 3.1 in Hallin et al. (1989) are thus fulfilled, and $J\left(\mathbf{u}_{1}, \ldots, \mathbf{u}_{l+1}\right)=u_{l, k} u_{j+1, l}$ is a score-generating function for $(n+1)^{-2}\left(\gamma_{j ; k, l}^{(n)}+m_{j^{*} ; k, l}^{(n)}\right)$. The corresponding "centered scores" (Proposition 2.1, same reference) are

$$
J_{j k l}^{*}\left(\mathbf{u}_{1}, \ldots, \mathbf{u}_{l+1}\right)=u_{1, k} u_{j+1, l}-\frac{1}{2}\left(u_{1, k}+u_{j+1, l}\right)-\frac{1}{4} .
$$

Proposition 2.2 (same reference as above) then entails the asymptotic joint binormality of $(n+1)^{-2}\left((n-I)^{\frac{1}{2}} \gamma_{j^{\prime} ; k^{\prime}, l^{\prime}}^{(n)},(n-I)^{\frac{1}{2}} \gamma_{j^{\prime} ; k^{\prime \prime}, l^{\prime \prime}}^{(n)}\right)^{\top}$, with mean $(0,0)^{\top}$ under $\mathcal{H}_{f}^{(n)}$, mean

$$
\left(\sum_{i=1}^{\min \left(j^{\prime}, \mathbf{K}\right)} \operatorname{tr}\left\{\mathbf{C}_{j^{\prime} k^{\prime} \nu^{\prime}}^{i}\left(\mathbf{A}_{i}+\mathbf{B}_{i}\right)\right\} \sum_{i=1}^{\min \left(j^{\prime \prime}, \mathbf{K}\right)} \operatorname{tr}\left\{\mathbf{C}_{j^{\prime \prime} k^{\prime \prime} l^{\prime \prime}}^{i}\left(\mathbf{A}_{i}+\mathbf{B}_{i}\right)\right\}\right)^{\top}
$$

under $\mathcal{K}_{f}^{(n)}(\mathbf{A}, \mathbf{B})$, and covariance matrix

$$
\left(\begin{array}{cc}
V_{j^{\prime} k^{\prime} l^{\prime}}^{2} & V_{12} \\
V_{12} & V_{j^{\prime \prime} k^{\prime \prime} l^{\prime \prime}}^{2}
\end{array}\right)
$$


under both, where $\mathrm{\kappa}=\max (p, q), \mathbf{A}_{i}=\mathbf{0}$ for $i=p+1, \ldots, \kappa, \mathbf{B}_{i}=\mathbf{0}$ for $i=q+1, \ldots, \kappa$, and with (all expectations below are taken under $\mathcal{H}_{f}^{(n)}$ )

$$
\begin{aligned}
\mathbf{C}_{j k l}^{i}= & \sum_{h=0}^{\kappa-i} \mathcal{E}\left\{J_{j k l}^{*}\left(\mathbf{F}\left(\mathbf{X}_{1}^{(n)}\right), \ldots, \mathbf{F}\left(\mathbf{X}_{l-1}^{(n)}\right)\right) \boldsymbol{\phi}\left(\mathbf{X}_{h+1}^{(n)}\right)\left(\mathbf{X}_{h+i+1}^{(n)}\right)^{\top}\right\} \\
= & \begin{cases}\mathcal{E}\left\{F_{k}\left(X_{1, k}^{(n)}\right) F_{l}\left(X_{j+1, l}^{(n)}\right) \mathbf{\phi}\left(\mathbf{X}_{1}^{(n)}\right)\left(\mathbf{X}_{j+1}^{(n)}\right)^{\top}\right\} & \text { if } i=j, \\
\mathbf{0} & \text { if } i \neq j\end{cases} \\
= & \begin{cases}\int F_{k}\left(x_{k}\right) \boldsymbol{\phi}(\mathbf{x}) d F(\mathbf{x}) \int F_{l}\left(y_{l}\right) \mathbf{y}^{\top} d F(\mathbf{y}) \text { if } i=j, \\
\mathbf{0} & \text { if } i \neq j,\end{cases} \\
V_{j k l}^{2}= & \mathcal{E}\left(J_{j k l}^{* 2}\left(\mathbf{F}\left(\mathbf{X}_{1}^{(n)}\right), \ldots, \mathbf{F}\left(\mathbf{X}_{l+1}^{(n)}\right)\right)\right. \\
& \left.+2 \sum_{h=1}^{l} J_{j k l}^{*}\left(\mathbf{F}\left(\mathbf{X}_{1}^{(n)}\right), \ldots, \mathbf{F}\left(\mathbf{X}_{l+1}^{(n)}\right)\right) J_{j k l}^{*}\left(\mathbf{F}\left(\mathbf{X}_{1+h}^{(n)}\right), \ldots, \mathbf{F}\left(\mathbf{X}_{l+1+h}^{(n)}\right)\right)\right) \\
= & \mathcal{E}\left(\left[F_{k}\left(X_{1, k}^{(n)}\right) F_{l}\left(X_{1+j, l}^{(n)}\right)-\frac{1}{2}\left\{F_{k}\left(X_{1, k}^{(n)}\right)+F_{l}\left(X_{1+j, l}^{(n)}\right)\right\}+\frac{1}{4}\right]^{2}\right) \\
= & \iint\left\{u v-\frac{1}{2}(u+v)+\frac{1}{4}\right\}^{2} d u d v=\frac{1}{144},
\end{aligned}
$$

and

$$
\begin{aligned}
& V_{12}=\mathcal{E}\left(J_{j^{\prime} k^{\prime} l^{\prime}}^{*}\left(\mathbf{F}\left(\mathbf{X}_{1}^{(n)}\right), \ldots, \mathbf{F}\left(\mathbf{X}_{l+1}^{(n)}\right)\right) J_{j^{\prime \prime} k^{\prime \prime} l^{\prime \prime}}^{*}\left(\mathbf{F}\left(\mathbf{X}_{1}^{(n)}\right), \ldots, \mathbf{F}\left(\mathbf{X}_{l+1}^{(n)}\right)\right)\right. \\
& +\sum_{h=1}^{l}\left\{J_{j^{\prime} k^{\prime} l^{\prime}}^{*}\left(\mathbf{F}\left(\mathbf{X}_{1}^{(n)}\right), \ldots, \mathbf{F}\left(\mathbf{X}_{l+1}^{(n)}\right)\right) J_{j^{\prime \prime} k^{\prime \prime} l^{\prime \prime}}^{*}\left(\mathbf{F}\left(\mathbf{X}_{1+h}^{(n)}\right), \ldots, \mathbf{F}\left(\mathbf{X}_{l+l+h}^{(n)}\right)\right)\right. \\
& \left.\left.+J_{j^{\prime} k^{\prime \prime} \nu^{\prime}}^{*}\left(\mathbf{F}\left(\mathbf{X}_{1+h}^{(n)}\right), \ldots, \mathbf{F}\left(\mathbf{X}_{l+1+h}^{(n)}\right)\right) J_{j^{\prime \prime} k^{\prime \prime} l^{\prime \prime}}^{*}\left(\mathbf{F}\left(\mathbf{X}_{1}^{(n)}\right), \ldots, \mathbf{F}\left(\mathbf{X}_{l+1}^{(n)}\right)\right)\right\}\right) \\
& = \begin{cases}\mathcal{E}\left(\left[F_{k^{\prime}}\left(X_{1, k^{\prime}}^{(n)}\right) F_{l^{\prime}}\left(X_{1+j, l^{\prime}}^{(n)}\right)-\frac{1}{2}\left\{F_{k^{\prime}}\left(X_{1, k^{\prime}}^{(n)}\right)+F_{l^{\prime}}\left(X_{1+j, l^{\prime}}^{(n)}\right)\right\}+\frac{1}{4}\right]\right. & \\
\left.\times\left[F_{k^{\prime \prime}}\left(X_{1, k^{\prime \prime}}^{(n)}\right) F_{l^{\prime \prime}}\left(X_{1+j, l^{\prime \prime}}^{(n)}\right)-\frac{1}{2}\left\{F_{k^{\prime \prime}}\left(X_{1, k^{\prime \prime}}^{(n)}\right)+F_{l^{\prime \prime}}\left(X_{1+j, l^{\prime \prime}}^{(n)}\right)\right\}+\frac{1}{4}\right]\right) & \text { if } j^{\prime}=j^{\prime \prime}=j, \\
0 & \text { if } j^{\prime} \neq j^{\prime \prime}\end{cases} \\
& =\left\{\left\{\int F_{k^{\prime}}\left(x_{k^{\prime}}\right) F_{k^{\prime \prime}}\left(x_{k^{\prime \prime}}\right) d F(\mathbf{x})-\frac{1}{4}\right\}\left\{\int F_{l^{\prime}}\left(x_{l^{\prime}}\right) F_{l^{\prime \prime}}\left(x_{l^{\prime \prime}}\right) d F(\mathbf{x})-\frac{1}{4}\right\} \begin{array}{c}
\text { if } j^{\prime}=j^{\prime \prime}=j, \\
\text { if } j^{\prime} \neq j^{\prime \prime} .
\end{array}\right.
\end{aligned}
$$

Proposition 2.1 follows.

The asymptotic distribution of $\Gamma_{i}^{n}$, as provided by Proposition 2.1, unfortunately cannot be used for inferential purposes, since the asymptotic covariance matrix (2.3) is not distribution-free, and clearly depends on the underlying joint density $f(\mathbf{x})$. Genuinely permutationally distribution-free rank statistics with asymptotic distribution-free unconditional distributions, however, can be obtained if conditional (permutational) covariances 
are substituted for the unconditional ones. The exact permutational covariance of vec $\Gamma_{i}^{(n)}$ is (for $n \geq 2 i$ )

$$
\begin{aligned}
\mathbf{W}_{i}^{*}= & \{n(n-1)(n-i)\}^{-1} \sum_{t_{1} \neq t_{2}}\left\{\mathbf{R}_{t_{1}}^{(n)}\left(\mathbf{R}_{t_{2}}^{(n)}\right)^{\top}\right\}^{\otimes 2} \\
+ & \left.2(n-2 i)\left\{n(n-1)(n-2)(n-i)^{2}\right\}^{-1} \sum_{t_{1} \neq t_{2} \neq t_{3}} \sum_{t_{t_{1}}}\left\{\mathbf{R}_{t_{2}}^{(n)}\right)^{\top}\right\} \otimes\left\{\mathbf{R}_{t_{3}}^{(n)}\left(\mathbf{R}_{t_{1}}^{(n)}\right)^{\top}\right\} \\
+ & \left\{n^{2}-n(2 i+3)+i^{2}+5 i\right\}\left\{n(n-1)(n-2)(n-3)(n-i)^{2}\right\}^{-1} \\
& \times \sum \sum_{t_{1} \neq t_{2} \neq i_{3} \neq t_{4}} \sum\left\{\mathbf{R}_{t_{1}}^{(n)}\left(\mathbf{R}_{t_{2}}^{(n)}\right)^{\top}\right\} \otimes\left\{\mathbf{R}_{t_{3}}^{(n)}\left(\mathbf{R}_{t_{4}}^{(n)}\right)^{\top}\right\}-\left(\operatorname{vec} \mathbf{m}_{i_{*}}^{(n)}\right)\left(\operatorname{vec} \mathbf{m}_{i *}^{(n)}\right)^{\top} .
\end{aligned}
$$

This exact, somewhat cumbersome form results from combinatorial arguments [see Lemma 2.1 in Hallin et al. (1989). In practice, it can be replaced by the following much simpler one, without affecting asymptotic results.

Proposition 2.2. Let

$$
\begin{aligned}
& \tilde{\mathbf{W}}_{i}^{*}=\{n(n-1)(n-i)\}^{-1} \\
& \times \sum_{l_{1} \neq f_{2}}\left[\mathbf{R}_{t_{1}}^{(n)}\left(\mathbf{R}_{t_{1}}^{(n)}\right)^{\top}-\frac{(n+1)^{2}}{4} \mathbf{1}\right] \otimes\left[\mathbf{R}_{t_{2}}^{(n)}\left(\mathbf{R}_{t_{2}}^{(n)}\right)^{\top}-\frac{(n+1)^{2}}{4} \mathbf{1}\right] .
\end{aligned}
$$

Then $\tilde{\mathbf{W}}_{i}^{*}-\mathbf{W}_{i}^{*}$ is o $o_{P}\left(n^{3}\right)$, and $\left(\operatorname{vec} \Gamma_{i}^{(n)}\right)^{\top}\left(\tilde{\mathbf{W}}_{i}^{*}\right)^{-1}\left(\operatorname{vec} \Gamma^{(n)}\right)$ is asymptotically chi-square (under $\left.\mathcal{H}^{(n)}\right)$, with $\mathrm{m}^{2}$ degrees of freedom, provided that

$$
\mathbf{V}=\int \mathbf{F}(\mathbf{x})\{\mathbf{F}(\mathbf{x})\}^{\top} d F(\mathbf{x})-\frac{1}{4}
$$

is a full-rank matrix.

Proof. It follows from Proposition 2.4(ii) in Hallin et al. (1989) that the permutational covariance matrix of $(n-i)^{\frac{1}{2}}(n+1)^{-2}$ vec $\Gamma_{i}^{(n)}$, viz. $(n-i)(n+1)^{-4} \mathbf{W}_{i}^{*}$, converges in probability to its asymptotical counterpart; $(n-i)(n+1)^{-4} \mathbf{W}_{i}^{*}-\mathbf{V}^{\otimes 2}=o_{P}(1)$ in (2.3). On the other hand, $(n-i)(n+1)^{-4} \tilde{\mathbf{W}}_{i}^{*}$ is the permutational mean of the (matrix of) linear serial multirank statistic(s) of order one

$$
(n-1)^{-1} \sum_{t=2}^{n}\left\{\frac{\mathbf{R}_{t}^{(n)}}{n+1}\left(\frac{\mathbf{R}_{t}^{(n)}}{n+1}\right)^{\top}-\frac{1}{4}\right\} \otimes\left\{\frac{\mathbf{R}_{t-1}^{(n)}}{n+1}\left(\frac{\mathbf{R}_{t-1}^{(n)}}{n+1}\right)^{\top}-\frac{1}{4}\right\},
$$

with a (matrix of) score-generating function(s) $J(u, v)=\left(\mathbf{u} \mathbf{u}^{\top}-\frac{1}{4}\right) \otimes\left(\mathbf{v} \mathbf{v}^{\top}-\frac{1}{4}\right)$ [this is a consequence of Proposition 3.1 in Hallin et al. (1989)]. Applying again Proposition 2.4 (same reference), this permutational mean converges in probability to its unconditional asymptotical counterpart:

$$
(n-i)(n+1)^{-4} \tilde{\mathbf{W}}_{i}^{*}-\mathbf{V}^{\otimes 2}=o_{P}(1) .
$$

It follows that $\tilde{\mathbf{W}}_{i}^{*}-\mathrm{W}_{i}^{*}$ is $o_{P}\left(n^{3}\right)$. Now, from Proposition 2.1 above, and provided that $\mathbf{V}$ (hence $\left.\mathbf{V}^{\otimes 2}\right)$ is of full rank, $Q_{i}^{(n)}=(n-i)(n+1)^{-4}\left(\operatorname{vec} \Gamma_{i}^{(n)}\right)^{\top}\left(\mathbf{V}^{\otimes 2}\right)^{-1}$ vec $\Gamma_{i}^{(n)}$ is asymptotically chi-square, under $\mathcal{H}^{(n)}$, with $m^{2}$ degrees of freedom. Since 
$\left(\operatorname{vec} \Gamma_{i}^{(n)}\right)^{\top}\left(\mathbf{W}_{i}^{*}\right)^{-1}\left(\operatorname{vec} \Gamma_{i}^{(n)}\right)$ and $\left(\operatorname{vec} \Gamma_{i}^{(n)}\right)^{\top}\left(\tilde{\mathbf{W}}_{i}^{*}\right)^{-1}\left(\operatorname{vec} \boldsymbol{\Gamma}_{i}^{(n)}\right)$ are asymptotically equivalent to $Q_{i}^{(n)}$, their asymptotic distribution is also chi-square - with however the additional property that they do not involve unknown quantities such as $V$. This completes the proof.

\section{STATISTICAL APPLICATIONS}

\subsection{Testing Randomness against Specified ARMA Dependence Alternatives.}

The distributional results of Section 2 of course can be exploited in the problem of testing the null hypothesis $\mathcal{H}^{(n)}$ of randomness. Two testing procedures are investigated here. The first one deals with the case of a specified alternative of ARMA dependence; the second one is a general, rank-based, portmanteau-type test, to be used against unspecified alternatives of serial dependence of order less than or equal to $\kappa$. Finally, a rank-based version of Tiao and Box's specification method is presented in Section 3.3.

If a particular local alternative of ARMA dependence, of the form (2.1), is considered, a "directional" test can be based on a linear combination of rank cross-covariances of the form

$$
T_{\mathrm{WW}}^{(n)}=\frac{\sum_{i=1}^{\kappa} \operatorname{tr}\left\{\mathbf{W}_{0}^{*-1} \boldsymbol{\Gamma}_{i}^{(n)}\left(\mathbf{A}_{i}+\mathbf{B}_{i}\right)^{\top}\right\}}{\left[\sum_{i=1}^{\boldsymbol{K}}\left\{\operatorname{vec} \mathbf{W}_{0}^{*-1}\left(\mathbf{A}_{i}+\mathbf{B}_{i}\right)\right\}^{\top} \mathbf{W}_{i}^{*}\left\{\operatorname{vec} \mathbf{W}_{0}^{*-1}\left(\mathbf{A}_{i}+\mathbf{B}_{i}\right)\right\}\right]^{\frac{1}{2}}},
$$

where

$$
\mathbf{W}_{0}^{*}=n^{-1} \sum_{t=1}^{n} \mathbf{R}_{t}^{(n)}\left(\mathbf{R}_{t}^{(n)}\right)^{\top}-\frac{(n+1)^{2}}{4} \mathbf{1}
$$

If necessary, $\tilde{\mathbf{W}}_{i}^{*}$ can be substituted for $\mathbf{W}_{i}^{*}$; as for $\mathbf{W}_{0}^{*}$, it is easy to see that $(n+1)^{-2} \mathbf{W}_{0}^{*}$ is a consistent, permutationally invariant estimator of $\mathbf{V}$; it is supposed here to be of full rank.

It follows from Proposition 2.1 that $T_{\mathrm{WW}}^{(n)}$ is exactly (i.e., permutationally) standardized, and hence asymptotically standard normal (under the assumptions of Proposition 2.1). Indeed, letting $\mathcal{E}_{*}$ denote permutational expectations,

$$
\begin{aligned}
\mathcal{E}_{*}\left\{\operatorname { t r } \mathbf { W } _ { 0 } ^ { * - 1 } \Gamma _ { i } ^ { ( n ) } \left(\mathbf{A}_{i}+\right.\right. & \left.\left.\mathbf{B}_{i}\right)^{\top}\right\}^{2} \\
& =\mathcal{E}_{*}\left\{\operatorname{vec}^{\top}\left(\mathbf{A}_{i}+\mathbf{B}_{i}\right) \operatorname{vec}\left(\mathbf{W}_{0}^{*-1} \Gamma_{i}^{(n)}\right) \operatorname{vec}^{\top}\left(\mathbf{W}_{0}^{*-1} \Gamma_{i}^{(n)}\right) \operatorname{vec}\left(\mathbf{A}_{i}+\mathbf{B}_{i}\right)\right\} \\
& =\left\{\operatorname{vec}^{\top}\left(\mathbf{A}_{i}+\mathbf{B}_{i}\right)\right\}\left(\mathbf{I} \otimes \mathbf{W}_{0}^{*-1}\right) \mathbf{W}_{i}^{*}\left(\mathbf{I} \otimes \mathbf{W}_{0}^{*-1}\right)\left\{\operatorname{vec}\left(\mathbf{A}_{i}+\mathbf{B}_{i}\right)\right\} \\
& =\operatorname{vec}^{\top}\left\{\mathbf{W}_{0}^{*-1}\left(\mathbf{A}_{i}+\mathbf{B}_{i}\right)\right\} \mathbf{W}_{i}^{*} \operatorname{vec}\left\{\mathbf{W}_{0}^{*-1}\left(\mathbf{A}_{i}+\mathbf{B}_{i}\right)\right\}
\end{aligned}
$$

Still on account of Proposition 2.1, the asymptotic power, under $\mathcal{K}_{f}^{(n)}(\mathbf{A}, \mathbf{B})$, of the test rejecting $\mathcal{H}^{(n)}$ whenever $T_{\mathrm{WW}}^{(n)}$ exceeds the standard normal $(1-\alpha)$-quantile $k_{1-\alpha}$ is

$$
\begin{aligned}
& \beta_{\mathrm{WW}}=1 \\
& -\Phi\left(k_{1-\alpha}-\frac{\sum_{i=1}^{\kappa} \operatorname{tr}\left[\mathcal{E}\left\{\mathbf{F}(\mathbf{X}) \boldsymbol{\phi}^{\top}(\mathbf{X})\right\}\left(\mathbf{A}_{i}+\mathbf{B}_{i}\right) \mathcal{E}\left\{\mathbf{F}(\mathbf{X}) \mathbf{X}^{\top}\right\}\left(\mathbf{A}_{i}+\mathbf{B}_{i}\right)^{\top} \mathbf{V}^{-1}\right]}{\left(\sum_{i=1}^{\mathrm{K}} \operatorname{tr}\left\{\left(\mathbf{A}_{i}+\mathbf{B}_{i}\right) \mathbf{V}\left(\mathbf{A}_{i}+\mathbf{B}_{i}\right)^{\top} \mathbf{V}^{-1}\right\}\right)^{\frac{1}{2}}}\right),
\end{aligned}
$$

where the expectations are computed under $\mathcal{H}_{f}^{(n)}$, and $\Phi$ denotes, as usual, the standard normal distribution function. 
The corresponding normal-theory parametric procedure relies (Hallin et al. 1989, Section 4.1) on the usual sample cross-covariance matrices

$$
\hat{\boldsymbol{\Gamma}}_{i}^{(n)}=(n-i)^{-1} \sum_{t=i+1}^{n} \mathbf{X}_{t}^{(n)}\left(\mathbf{X}_{t-i}^{(n)}\right)^{\top}
$$

[if the assumption that $\mathcal{E} \mathbf{X}_{t}=\mathbf{0}$ is not to be made, the observations in (3.4) and in the subsequent formulas have to be centered about their mean] and rejects the null hypothesis of (supposedly full-rank Gaussian) randomness whenever

$$
T_{\mathrm{G}}^{(n)}=\frac{\sum_{i=1}^{\mathrm{K}}(n-i)^{\frac{1}{2}} \operatorname{tr}\left\{\left(\hat{\boldsymbol{\Gamma}}_{0}^{(n)}\right)^{-1} \hat{\boldsymbol{\Gamma}}_{i}^{(n)}\left(\mathbf{A}_{i}+\mathbf{B}_{i}\right)^{\top}\right\}}{\left.\left\{\sum_{i=1}^{\mathrm{K}}\left\{\operatorname{vec}\left(\hat{\boldsymbol{\Gamma}}_{0}^{(n)}\right)^{-1}\left(\mathbf{A}_{i}+\mathbf{B}_{i}\right)\right\}^{\top} \hat{\boldsymbol{\Gamma}}_{0}^{(n)}\right)^{\otimes 2}\left\{\operatorname{vec}\left(\hat{\boldsymbol{\Gamma}}_{0}^{(n)}\right)^{-1}\left(\mathbf{A}_{i}+\mathbf{B}_{i}\right)\right\}\right\}^{\frac{1}{2}}}>k_{1-\alpha} .
$$

The resulting asymptotic power, still under $\mathcal{K}_{f}^{\top}(\mathbf{A}, \mathbf{B})$, is

$$
\boldsymbol{\beta}_{\mathrm{G}}=1-\Phi\left(k_{1-\alpha}-\left\{\sum_{i=1}^{\mathrm{K}} \operatorname{tr}\left\{\left(\mathbf{A}_{i}+\mathbf{B}_{i}\right) \mathbf{Z}\left(\mathbf{A}_{i}+\mathbf{B}_{i}\right)^{\top} \mathbf{Z}^{-1}\right\}\right\}^{\frac{1}{2}}\right)
$$

The asymptotic relative efficiency of the Wald-Wolfowitz test based on $T_{\mathrm{W} w}^{(n)}$ with respect to the Gaussian one based on $T_{\mathrm{G}}^{(n)}$ accordingly is

$$
\begin{aligned}
& \operatorname{ARE}\left(T_{W W}^{(n)} ; T_{\mathrm{G}}^{(n)}\right) \\
& =\frac{\left(\sum_{i=1}^{\mathrm{K}} \operatorname{tr}\left[\mathcal{E}\left\{\mathbf{F}(\mathbf{X}) \boldsymbol{\phi}^{\top}(\mathbf{X})\right\}\left(\mathbf{A}_{i}+\mathbf{B}_{i}\right) \mathcal{E}\left\{\mathbf{F}(\mathbf{X}) \mathbf{X}^{\top}\right\}\left(\mathbf{A}_{i}+\mathbf{B}_{i}\right)^{\top} \mathbf{V}^{-1}\right]\right)^{2}}{\left(\sum_{i=1}^{\kappa} \operatorname{tr}\left\{\left(\mathbf{A}_{i}+\mathbf{B}_{i}\right) \mathbf{V}\left(\mathbf{A}_{i}+\mathbf{B}_{i}\right)^{\top} \mathbf{V}^{-1}\right\}\right)\left(\sum_{i=1}^{\kappa} \operatorname{tr}\left\{\left(\mathbf{A}_{i}+\mathbf{B}_{i}\right) \mathbf{Z}\left(\mathbf{A}_{i}+\mathbf{B}_{i}\right)^{\top} \mathbf{Z}^{-1}\right\}\right)}
\end{aligned}
$$

The matrices $\mathcal{E}\left\{\mathbf{F}(\mathbf{X}) \mathbf{X}^{\top}\right\}, \mathcal{E}\left\{\mathbf{F}(\mathbf{X}) \boldsymbol{\phi}^{\top}(\mathbf{X})\right\}$ and $\mathbf{V}=\mathcal{E}\left\{\mathbf{F}(\mathbf{X}) \mathbf{F}^{\top}(\mathbf{X})\right\}-\frac{1}{4}$ in general are not easy to compute. If however $f$ itself is Gaussian, with covariance matrix $\boldsymbol{Z}=\left(\sigma_{i j}\right)$, then $\boldsymbol{\phi}(\mathbf{X})=\mathbf{Z}^{-1} \mathbf{X}$,

$$
\mathcal{E}\left\{F_{i}\left(X_{i}\right) X_{j}\right\}=\frac{\sigma_{i j}}{\sigma_{i i}} \mathcal{E}\left\{F_{i}\left(X_{i}\right) X_{i}\right\}=\frac{\sigma_{i j}}{\sigma_{i i}^{\frac{1}{2}}} \int_{0}^{1} u \Phi^{-1}(u) d u=\frac{\sigma_{i j}}{\sigma_{i i}^{\frac{1}{2}}}(4 \pi)^{-\frac{1}{2}},
$$

which implies

$$
\mathcal{E}\left\{\mathbf{F}(\mathbf{X}) \mathbf{X}^{\top}\right\}=\operatorname{diag}(2 \sqrt{\pi} \sigma)^{-1} \mathbf{Y},
$$

and

$$
\mathcal{E}\left\{\mathbf{F}(\mathbf{X}) \boldsymbol{\phi}^{\top}(\mathbf{X})\right\}=\mathcal{E}\left\{\mathbf{F}(\mathbf{X}) \mathbf{X}^{\top}\right\} \mathbf{Y}^{-1}=\operatorname{diag}(2 \sqrt{\pi} \sigma)^{-1},
$$

where $\operatorname{diag}(2 \sqrt{\pi} \sigma)^{-1}$ stands for the diagonal matrix with $i$ th diagonal element $\left(2 \sqrt{\pi} \sigma_{i i}^{\frac{1}{2}}\right)^{-1}$. If moreover $\mathbf{Z}=\sigma^{2} I$, then

$$
\mathbf{V}=\mathcal{E}\left\{\mathbf{F}(\mathbf{X}) \mathbf{F}^{\top}(\mathbf{X})\right\}-\frac{1}{4}=\left[\int u^{2} d u-\frac{1}{4}\right] \mathbf{I}=\frac{\mathbf{I}}{12}
$$

and (3.7) reduces to $(3 / \pi)^{2}$, which is exactly the ARE, under Gaussian assumptions, of the univariate Wald-Wolfowitz test against serial dependence with respect to its parametric counterpart, based on usual sample autocorrelations (see, e.g., Hallin et al. 1985). 


\subsection{A Wald-Wolfowitz Multivariate Portmanteau Test.}

Specified alternatives such as (2.1) seldom are considered in practice. In the case of an omnibus alternative of $\operatorname{ARMA}(p, q)$ dependence, with unspecified coefficients $\boldsymbol{A}_{i}$ and $B_{i}$ but with orders $p$ and $q$ smaller than $\kappa$, one might like to consider the following rank-based portmanteau procedure, which consists in rejecting $\mathcal{H}^{(n)}$ whenever

$$
Q_{\mathrm{WW}}^{(n)}=\sum_{i=1}^{\mathrm{K}}\left(\operatorname{vec} \Gamma_{i}^{(n)}\right)^{\top}\left\{\tilde{\mathbf{W}}_{i}^{*}\right\}^{-1}\left(\operatorname{vec} \Gamma_{i}^{(n)}\right)>\chi_{\mathrm{km} m^{2} ; 1-\alpha}^{2},
$$

where $\chi_{1 m^{2} ; 1-\alpha}^{2}$ denotes the $(1-\alpha)$-quantile of a chi-square variable with $\mathrm{km}^{2}$ degrees of freedom. The corresponding Gaussian portmanteau test relies on

$$
Q_{\mathrm{G}}^{(n)}=\sum_{i=1}^{\kappa}(n-i)\left(\operatorname{vec} \hat{\boldsymbol{\Gamma}}_{i}^{(n)}\right)^{\top}\left\{\left(\hat{\boldsymbol{\Gamma}}_{0}^{(n)}\right)^{\otimes 2}\right\}^{-1}\left(\operatorname{vec} \hat{\boldsymbol{\Gamma}}_{i}^{(n)}\right)
$$

which is also (Chiturri 1976) asymptotically chi-square, under the hypothesis of Gaussian, full-rank white noise, still with $\mathrm{km}^{2}$ degrees of freedom.

The asymptotic powers and relative efficiencies of these two portmanteau procedures under $\mathcal{K}_{f}^{(n)}(\mathbf{A}, \mathbf{B})$ depend on the noncentrality parameters

$$
\begin{aligned}
& \lambda_{\mathrm{WW}}^{2}(\mathbf{A}, \mathbf{B} ; f) \\
& =\sum_{i=1}^{\kappa} \operatorname{vec}^{\top}\left(\mathbf{A}_{i}+\mathbf{B}_{i}\right) \\
& \times\left[\mathcal{E}\left\{\mathbf{X} \mathbf{F}^{\top}(\mathbf{X})\right\} \otimes \mathcal{E}\left\{\boldsymbol{\phi}(\mathbf{X}) \mathbf{F}^{\top}(\mathbf{X})\right\}\right]\left(\mathbf{V}^{-1}\right)^{\otimes 2}\left[\mathcal{E}\left\{\mathbf{F}(\mathbf{X}) \mathbf{X}^{\top}\right\} \otimes \mathcal{E}\left\{\mathbf{F}(\mathbf{X}) \boldsymbol{\phi}^{\top}(\mathbf{X})\right\}\right] \\
& \times \operatorname{vec}\left(\mathbf{A}_{i}+\mathbf{B}_{i}\right) \\
& =\sum_{i=1}^{\mathbf{K}} \operatorname{tr}\left[\mathbf{V}^{-1} \mathcal{E}\left\{\mathbf{F}(\mathbf{X}) \boldsymbol{\phi}^{\top}(\mathbf{X})\right\}\left(\mathbf{A}_{i}+\mathbf{B}_{i}\right) \mathcal{E}\left\{\mathbf{F}(\mathbf{X}) \mathbf{X}^{\top}\right\} \mathbf{V}^{-1}\right. \\
& \left.\times \mathcal{E}\left\{\mathbf{X F}^{\top}(\mathbf{X})\right\}\left(\mathbf{A}_{i}+\mathbf{B}_{i}\right)^{\top} \mathcal{E}\left\{\boldsymbol{\phi}(\mathbf{X}) \mathbf{F}^{\top}(\mathbf{X})\right\}\right] \\
& =\sum_{i=1}^{\mathbf{K}} \operatorname{vec}^{\top}\left(\mathbf{A}_{i}+\mathbf{B}_{i}\right)\left[\mathcal{E}\left\{\mathbf{F}(\mathbf{X}) \mathbf{X}^{\top}\right\} \mathbf{V}^{-1} \mathcal{E}\left\{\mathbf{X F}^{\top}(\mathbf{X})\right\}\right] \\
& \otimes\left[\mathcal{E}\left\{\boldsymbol{\phi}(\mathbf{X}) \mathbf{F}^{\top}(\mathbf{X})\right\} \mathbf{V}^{-1} \mathcal{E}\left\{\mathbf{F}(\mathbf{X}) \boldsymbol{\phi}^{\top}(\mathbf{X})\right\}\right] \operatorname{vec}\left(\mathbf{A}_{i}+\mathbf{B}_{i}\right)
\end{aligned}
$$

and

$$
\begin{aligned}
\lambda_{\mathbf{G}}^{2}(\mathbf{A}, \mathbf{B} ; f) & =\sum_{i=1}^{\kappa} \operatorname{vec}^{\top}\left(\mathbf{A}_{i}+\mathbf{B}_{i}\right)(\mathbf{Z} \otimes \mathbf{I})\left(\mathbf{Z}^{-1}\right)^{\otimes 2}(\mathbf{\Psi} \otimes \mathbf{I}) \operatorname{vec}\left(\mathbf{A}_{i}+\mathbf{B}_{i}\right) \\
& =\sum_{i=1}^{\kappa} \operatorname{tr}\left\{\mathbf{\Psi}^{-1}\left(\mathbf{A}_{i}+\mathbf{B}_{i}\right) \mathbf{Z}\left(\mathbf{A}_{i}+\mathbf{B}_{i}\right)^{\top}\right\} \\
& =\sum_{i=1}^{\kappa} \operatorname{vec}^{\top}\left(\mathbf{A}_{i}+\mathbf{B}_{i}\right)\left(\mathbf{\Sigma} \otimes \mathbf{Z}^{-1}\right) \operatorname{vec}\left(\mathbf{A}_{i}+\mathbf{B}_{i}\right)
\end{aligned}
$$


The asymptotic relative efficiency of the Wald-Wolfowitz procedure with respect to the Gaussian one, under $\mathcal{K}_{f}^{(n)}(\mathbf{A}, \mathbf{B})$, accordingly is

$$
\operatorname{ARE}\left(Q_{\mathrm{WW}}^{(n)} ; Q_{\mathrm{G}}^{(n)}\right)=\frac{\lambda_{\mathrm{WW}}^{2}(\mathbf{A}, \mathbf{B} ; f)}{\lambda_{\mathrm{G}}^{2}(\mathbf{A}, \mathbf{B} ; f)} .
$$

Even under Gaussian assumptions, there is no guarantee that this ARE will be smaller than one; if, however, $F$ is Gaussian with diagonal covariance matrix $\sigma^{2} I$, (3.10) again reduces to $9 / \pi^{2}$.

\subsection{A Rank-Based Version of Tiao and Box's Tentative Specification Method.}

Tiao and Box (1981) suggest basing a tentative specification of multivariate ARMA models on the following device. Denoting by $\hat{\rho}_{i ; k, l}^{(n)}$ the sample cross-correlation

$$
\sum_{t=i+l}^{n} X_{t, k}^{(n)} X_{t-i, l}^{(n)}\left(\sum_{l}\left(X_{t, k}^{(n)}\right)^{2} \sum_{t}\left(X_{t, l}^{(n)}\right)^{2}\right)^{-\frac{1}{2}}
$$

they propose considering, for each lag $i=1,2, \ldots$, a matrix where element $(k, l)$ is replaced with

a plus sign if $\hat{\rho}_{i, k, l}^{(n)}$ is greater than $2 n^{-\frac{1}{2}}$,

a minus sign if $\hat{\rho}_{i ; k, l}^{(n)}$ is less than $-2 n^{-\frac{1}{2}}$,

a dot if $\hat{\rho}_{i ; k, l}^{(n)}$ lies in $\left[ \pm 2 n^{-\frac{1}{2}}\right]$.

These indicator symbols of course cannot be considered as resulting from formal (5\%) significance tests, but (quoting the authors) "as a rather crude signal-to-noise ratio guide (...) providing useful and assimilable indicators of the general correlation pattern."

From Proposition 2.1, it follows that the rank-based cross-covariance coefficients $\gamma_{i ; k, l}^{(n)}$ are asymptotically normal under $\mathcal{H}^{(n)}$, with mean zero and variance $(n+1)^{4} / 144(n-i)$. Accordingly, this widely used device of Tiao and Box can be modified in an obvious way, substituting rank-based cross-covariances for sample autocorrelations, and replacing $\gamma_{i ; k, l}^{(n)}$ in $\Gamma_{i}^{(n)}$ with

a plus sign if $\gamma_{i ; k, l}^{(n)}$ is greater than $(n+1)^{2} / 6(n-i)^{\frac{1}{2}}$,

a minus sign if $\gamma_{i ; k, l}^{(n)}$ is less than $-(n+1)^{2} / 6(n-i)^{\frac{1}{2}}$,

a dot if $\gamma_{i, k, l}^{(n)}$ lies in $\left[ \pm(n+1)^{2} / 6(n-i)^{\frac{1}{2}}\right]$.

This Wald-Wolfowitz rank-based version of Tiao and Box's procedure is likely to be more reliable under non-Gaussian conditions, and more robust against gross error. Contrary to the methods described in Sections 3.1 and 3.2 above, its practical implementation is quite straightforward.

\section{ACKNOWLEDGEMENT}

The authors gratefully acknowledge the very careful reading of the manuscript by an Associate Editor and a referee, which significantly improved the final presentation of the paper.

\section{REFERENCES}

Bartels, R. (1982). The rank version of von Neumann's ratio test for randomness. J. Amer. Statist. Assoc., 77 , $40-46$. 
Chan, N.H., and Tran, L.T. (1992). Nonparametric tests for serial dependence. J. Time Ser. Anal., 13, 19-28.

Chiturri, R.V. (1976). Distribution of multivariate white noise autocorrelations. J. Amer. Statist. Assoc., 71, 223-226.

Dufour, J.-M. (1981). Rank tests for serial dependence. J. Time Ser. Anal., 2, 117-128.

Dufour, J.-M., and Roy, R. (1985). Some robust exact results on sample autocorrelations and tests of randomness. J. Econometrics, 29, 257-273.

Dufour, J.-M., and Roy, R. (1986). Generalized portmanteau statistics and tests of randomness. Comm. Statist. Theory Methods, 15, 2953-2972.

Hallin, M., Ingenbleek, J.-Fr., and Puri, M.L. (1985). Linear serial rank tests for randomness against ARMA alternatives. Ann. Statist., 13, 1156-1181.

Hallin, M., Ingenbleek, J.-Fr., and Puri, M.L. (1987). Linear and quadratic rank tests for randomness against serial dependence. J. Time Ser. Anal., 8, 409-424.

Hallin, M., Ingenbleek, J.-Fr., and Puri, M.L. (1989). Asymptotically most powerful rank tests for multivariate randomness against serial dependence. J. Multivariate Anal., 30, 34-71.

Hallin, M., and Mélard, G. (1988). Rank-based tests for randomness against first-order serial dependence. $J$. Amer. Statist. Assoc., 83, 1117-1128.

Hallin, M., and Puri, M.L. (1988). Optimal rank-based procedures for time series analysis: Testing an ARMA model against other ARMA models. Ann. Statist., 16, 402-432.

Hallin, M., and Puri, M.L. (1991). Times series analysis via rank-order theory: Signed rank tests for ARMA models. J. Multivariate Anal., 39, 1-29.

Hallin, M., and Puri, M.L. (1992). Rank tests for time series analysis: A survey. New Directions in Time Series Analysis (D. Brillinger et al., eds.), Springer-Verlag, 111-153.

Hallin, M., and Puri, M.L. (1994). Aligned rank tests for linear models with autocorrelated error terms. $J$. Multivariate Anal., 50, 175-237.

Knoke, D.J. (1977). Testing for randomness against autocorrelation: Alternative tests. Biometrika, 64, 523-529.

Skaug, H.J., and Tjøstheim, D. (1993). Nonparametric tests of serial independence. Developments in Time Series Analysis, Volume in Honour of M.B. Priestley (T. Subba Rao, ed), Chapman and Hall, 207-229.

Tiao, G.C., and Box, G.E.P. (1981). Modeling multiple time series with applications. J. Amer. Statist. Assoc., $76,802-816$.

Wald, A., and Wolfowitz, J. (1943). An exact test for randomness in the nonparametric case based on serial correlation. Ann. Math. Statist., 14, 378-388.

Received 15 November 1993

Revised 26 May 1994

Accepted 13 June 1994
Institut de Statistique et Département de Mathématique Universite Libre de Bruxelles Campus de la Plaine C.P. 210

B 1050 Bruxelles

Belgium

Department of Mathematics Indiana University

Bloomington, Indiana U.S.A. 47405 